\title{
Author Correction: Radiology: Handy magnetic resonance coils
}

Elmar Laistler and Ewald Moser

Correction to: Nature Biomedical Engineering https://doi.org/10.1038/s41551-018-0278-y, published online 9 August 2018.

In the version of this News and Views article originally published, the author name, title and year were incorrect in ref. ${ }^{14}$ and should have read "Yang, X. et al. Coaxial cable magnetic resonance image (MRI) coil. US patent US9678180B2 (2017)." This error has now been corrected. 